\title{
A Noninvasive Method to Identify Ineffective Triggering in Patients with Noninvasive Pressure Support Ventilation
}

\author{
Antoine Cuvelier ${ }^{a}$ Linda Achour ${ }^{a}$ b Herinaina Rabarimanantsoa ${ }^{b}$ \\ Christophe Letellier $^{\mathrm{b}}$ Jean-François Muir ${ }^{\mathrm{a}}$ Brigitte Fauroux ${ }^{\mathrm{c}}$ \\ a Pulmonary and Intensive Care Department, Rouen University Hospital \& UPRES EA 3830 (IFR MP23), Institute for \\ Biomedical Research, and ${ }^{\mathrm{b} C O R I A}$ UMR 6614, University of Rouen, Rouen, and ' AP-HP, Hôpital Armand Trousseau, \\ Pediatric Pulmonary Department and Research Unit Inserm UMR S-718, Université Pierre et Marie Curie-Paris 6, \\ Paris, France
}

\section{Key Words}

Chronic respiratory failure $\cdot$ Cystic fibrosis $\cdot$ Noninvasive mechanical ventilation - Noninvasive monitoring •

Patient-ventilator asynchrony

\begin{abstract}
Background: Ineffective inspiratory triggering efforts are a major cause of poor patient-ventilator interactions during mechanical ventilation, but their routine identification requires the insertion of an esophageal catheter. Objectives: We developed a mathematical analysis of ventilatory tracings recorded under noninvasive pressure ventilation in order to identify ineffective triggering efforts and their consequences without recording esophageal pressure. Methods: We assessed 2,183 cycles from 44 pressure support tracings in 14 children with cystic fibrosis treated by noninvasive home ventilation. Airway pressure, flow and esophageal pressure time series were visually analyzed and manually counted. Airway pressure versus time and flow versus time were then analyzed using a dedicated algorithm written by us. Esophageal pressure was only used for validation. $\boldsymbol{R e}$ sults: A mathematical treatment of flow time series allowed us to draw phase portraits that had specific periodic trajec-
\end{abstract}

\section{KARGER}

Fax +41613061234 E-Mail karger@karger.ch www.karger.com
(C) 2009 S. Karger AG, Basel 0025-7931/10/0803-0198\$26.00/0

Accessible online at:

www.karger.com/res tories for triggered ventilatory cycles and ineffective triggering efforts. From flow and pressure tracings, our algorithm correctly identified $100 \%$ of triggered cycles and 53/56 (94.6\%) of ineffective triggering efforts. Ineffective triggering was associated with a significant reduction in minute ventilation, inspiratory flows and a significant increase in inspiratory efforts. Conclusions: A noninvasive analysis of flow and airway pressure can reliably identify ineffective triggering efforts during noninvasive pressure support ventilation. This approach may be a valuable tool for evaluating patient-ventilator interactions and their consequences during long-term recordings.

Copyright $\odot 2009$ S. Karger AG, Basel

\section{Introduction}

Poor patient-ventilator interactions and especially ineffective inspiratory triggering efforts (ITE) may occur during noninvasive pressure support ventilation (PSV) in patients with cystic fibrosis (CF) [1, 2], in COPD patients with lung inflation and intrinsic positive end-expiratory pressure (PEEP) $[3,4]$ and in patients with various other pulmonary diseases [5]. ITE under PSV are more fre- 
quent during sleep $[6,7]$ and when increasing the level of ventilatory assistance $[8,9]$. ITE during noninvasive ventilation are associated with poorer gas exchange during sleep [10] and sleep fragmentation [7, 11]. Moreover, ITE may be a cause of PSV intolerance and failure in critically ill patients 'fighting' against their ventilator. Otherwise, the incidence of ITE and its impact on PSV efficacy and comfort remain unknown.

A simple noninvasive method to detect ITE during PSV is therefore highly desirable. Esophageal pressure $\left(\mathrm{P}_{\mathrm{es}}\right)$ monitoring is commonly used as the standard for detecting the onset of inspiratory efforts, although diaphragmatic electromyography has recently been used by some authors [12]. This latter technique requires esophageal or surface electrodes and cannot be performed on a routine basis during sleep or for domiciliary assessments. Consequently, most studies on ITE have assessed $\mathrm{P}_{\mathrm{es}}$ and/ or transdiaphragmatic pressure variations to identify the relationship between individual inspiratory efforts and ventilator triggering. However, esophageal recordings are not practical as a routine measure in most centers and are inadequate for prolonged or long-term assessments in patients treated with home ventilation.

On the other hand, manual scoring of ITE is timeconsuming. With the increasing interest in patient-ventilator interactions during noninvasive PSV, the development of an automatic system to detect and monitor ITE would be a welcome advance in the management of patients with an incomplete response to ventilatory treatment. We thus developed an algorithm that integrates airway pressure $\left(\mathrm{P}_{\mathrm{aw}}\right)$ and flow variations to automatically detect ITE in real time. The objective of our study was to validate this automatic noninvasive method by comparing it with the manual detection of ITE using conventional $\mathrm{P}_{\mathrm{es}}$ recordings. We also evaluated the modification of the breathing pattern associated with ITE occurrence.

\section{Patients and Methods}

\section{Patients}

We retrospectively analyzed tracings that were routinely recorded under spontaneous breathing and under mechanical ventilation during the initiation of noninvasive PSV in children (6 males, 8 females) with advanced stable CF lung disease and who had no previous experience with noninvasive ventilation. These tracings were obtained during two previously published studies $[13,14]$, in which written approval was obtained from the parents and the patients. The current analysis was approved by the Ethical Committee of Saint Antoine Hospital (Paris, France).
The patients were started on home ventilation $7.4 \pm 5.7$ months earlier because of daytime hypercapnia (mean $\mathrm{PaCO}_{2}=$ $58.5 \pm 12.1 \mathrm{~mm} \mathrm{Hg}$ ) and symptoms of nocturnal hypoventilation $[2,13]$. At the time of the recordings, their mean age was $15.6 \pm$ 3.1 years and their mean BMI was $16.7 \pm 1.5$. Mean forced vital capacity was $45.6 \pm 9.8 \%$ of predicted values and mean forced expiratory volume in $1 \mathrm{~s}$ was $32.0 \pm 4.8 \%$ of predicted values. Mean $\mathrm{PaO}_{2}$ was $60.7 \pm 8.6 \mathrm{~mm} \mathrm{Hg}$ and mean $\mathrm{PaCO}_{2}$ was $42.3 \pm$ $3.3 \mathrm{~mm} \mathrm{Hg}$ under room air. Recordings were obtained during spontaneous breathing and during PSV. Additional oxygen was added during spontaneous breathing and PSV to maintain $\mathrm{SpO}_{2}$ $>92 \%$.

\section{Measurements and PSV Settings}

Ventilatory data were recorded for $5 \mathrm{~min}$ after a stable breathing pattern lasting $15 \mathrm{~min}$. Flow was measured with a pneumotachograph (No. 2; Fleisch, Lausanne, Switzerland) located between the nasal mask and the expiratory valve and connected to a differential pressure transducer (MP 45, Validyne Corp., Northridge, Calif., USA). Tidal volume (VT) was obtained by integrating the flow signal. $\mathrm{SpO}_{2}$, respiratory rate (RR) and cardiac frequency were continuously recorded during the study. $\mathrm{P}_{\mathrm{es}}$ was measured using a 2.1-mm external diameter catheter (Gaeltec, Dunvegan, UK) that was inserted pernasally into one nostril after the administration of topical anesthesia. Appropriate placement of the $\mathrm{P}_{\mathrm{es}}$ transducer was assessed as previously described [15]. The signals were digitized and sampled at $200 \mathrm{~Hz}$ for analysis using an MP100 acquisition system (Biopac Systems, Goleta, Calif., USA).

The patients were studied in the semirecumbent position and were asked to breathe quietly throughout the recordings. PSV was delivered either with a VS Integra ${ }^{\circledR}$ (ResMed, France) or an On$\mathrm{yx}^{\circledR}$ (Covidien, France) ventilator through a tight-fitting nasal mask (Respironics, Murrysville, Pa., USA) using the manufacturer's recommended single branch circuit with an expiratory valve. Inspiratory positive airway pressure (IPAP) was progressively increased to the level associated with minimal $\mathrm{P}_{\mathrm{es}}$ variations. Mean IPAP was $15.9 \pm 3.0 \mathrm{~cm} \mathrm{H}_{2} \mathrm{O}$ and no PEEP was added. The backup frequency was set at 12 breaths/min. The inspiratory trigger was set at the most sensitive value without autotriggering and expiratory cycling was set at $35 \%$ of peak inspiratory flow. The pressure slope was set at the steepest value tolerated by the patient. The size of the mask was chosen to be the most comfortable as possible and great care was taken to obtain ventilation without unintentional leaks.

Manual Analysis of Tracings

Two independent investigators (L.A. and C.L.) analyzed the spontaneous breathing and ventilatory tracings. Both operators were blinded to the algorithm results and were not aware of the other scoring. After identifying artifacts (cough or esophageal spasms), the remaining cycles were used to calculate breathing patterns and inspiratory efforts. Similarly for triggered and nontriggered cycles, inspiratory time (TI), expiratory time (TE), relative inspiratory time $\left(\mathrm{TI} / \mathrm{T}_{\text {tot }}\right)$, VT and mean inspiratory flow (VT/ $\mathrm{TI})$ were measured from the flow signal. Minute expiratory ventilation (VE) and RR were values from all individual triggered or nontriggered breaths extrapolated to $1 \mathrm{~min}$. Inspiratory muscle output was estimated from $\mathrm{P}_{\mathrm{es}}$ swings and the esophageal pressure-time product $\left(\mathrm{PTP}_{\mathrm{es}}\right)$. $\mathrm{PTP}_{\mathrm{es}} /$ breath was obtained by measuring the area under the $\mathrm{P}_{\mathrm{es}}$ signal from the onset of the negative 
deflection to the return to baseline (fig. 1). This approach was slightly different from the standard method that consists of measuring the area under the $\mathrm{P}_{\mathrm{es}}$ signal from the onset of a negative deflection until its following minimal value. Nevertheless, our method could better avoid aberrant measures due to a difficult automatic recognition that sometimes arises when this minimal $\mathrm{P}_{\mathrm{es}}$ signal is irregular. It led to nearly double PTP values compared to the standard method, but this had no consequence on the final message since it did not affect the relative order of PTP values obtained in this way and the comparability between all the cycles that were studied in the same way. ITE were defined by a $>1 \mathrm{~cm}$ $\mathrm{H}_{2} \mathrm{O}$ decrease in $\mathrm{P}_{\mathrm{es}}$ without subsequent delivery of IPAP [8]. For each ventilatory cycle, including ITE, the mean airway pressuretime product $\left(\mathrm{PTP}_{\mathrm{aw}}\right)$ was calculated as the integral of $\mathrm{P}_{\mathrm{aw}}$ during the trigger time delay (fig. 1). In this study, the trigger time delay was defined as the duration between the onset of the drop in pressure until its return to baseline (fig. 1).

\section{Automatic Analysis of Tracings}

The ventilatory tracings were then analyzed using a two-step automatic process. To obtain a global aspect of patient-ventilator interactions and especially ITE, phase portraits were first reconstructed from the sole flow time series in the tracings, using time delay coordinates [16]. A phase portrait is a geometric representation of the evolution of the patient-ventilator system as a trajectory in a space spanned by any dynamical variables such as flow, $\mathrm{P}_{\mathrm{aw}}$ or $\mathrm{P}_{\mathrm{es}}[16]$. One of the results of the nonlinear dynamical system theory is the possibility to build this trajectory from a single time series using delay coordinates [17]. For instance, a plane projection of a reconstructed space can be spanned by the flow at time $t+\tau$ plotted versus the flow at time $t$ where $\tau$ is a time delay. The curve obtained in this way presents a structure that directly depends on the time evolution of the patient-ventilator system. Thus, different patient-ventilator interactions were associated with different curve shapes. Typically, each respiratory cycle is represented by a loop in the phase space (fig. $2 \mathrm{a}$ ). Such a loop starts roughly at the origin $[$ flow $(t)=0$, flow $(t+\tau)=0]$ with the beginning of the inspiration. A quick jump to an upper plateau corresponds to the rise in pressure. At the end of inspiration, the trajectory switches to a lower plateau associated with expiration, followed by a return to the origin, and so on for further cycles [16].

The ventilatory tracings were then analyzed with a dedicated algorithm written by L.A. and C.L. in FORTRAN that was designed to identify ITE from the rate of change of the flow signal and the corresponding maximal $\mathrm{P}_{\mathrm{aw}}$, that is, without the aid of $\mathrm{P}_{\mathrm{es}}$ variation curves. Once the rate of change of the airflow was greater than 1 liter/min during $20 \mathrm{~ms}$ [16], it was checked whether the inspiratory pressure was greater than the pressure threshold value or not. If not, an ITE was identified. The algorithm detected and quantified the occurrence of ITE based on features as described by Giannouli et al. [9]. Breathing patterns were also computed with this algorithm. In our automatic analysis, ITE were defined as any inspiratory effort producing a flow rate of change greater than a preset value associated with a pressure less than a threshold defined as (IPAP + PEEP)/2. In the present case, since there was no PEEP, this meant that the threshold was actually IPAP/2. The number of ITE automatically calculated for all tracings was then compared to the number of ITE manually counted from the same tracings.

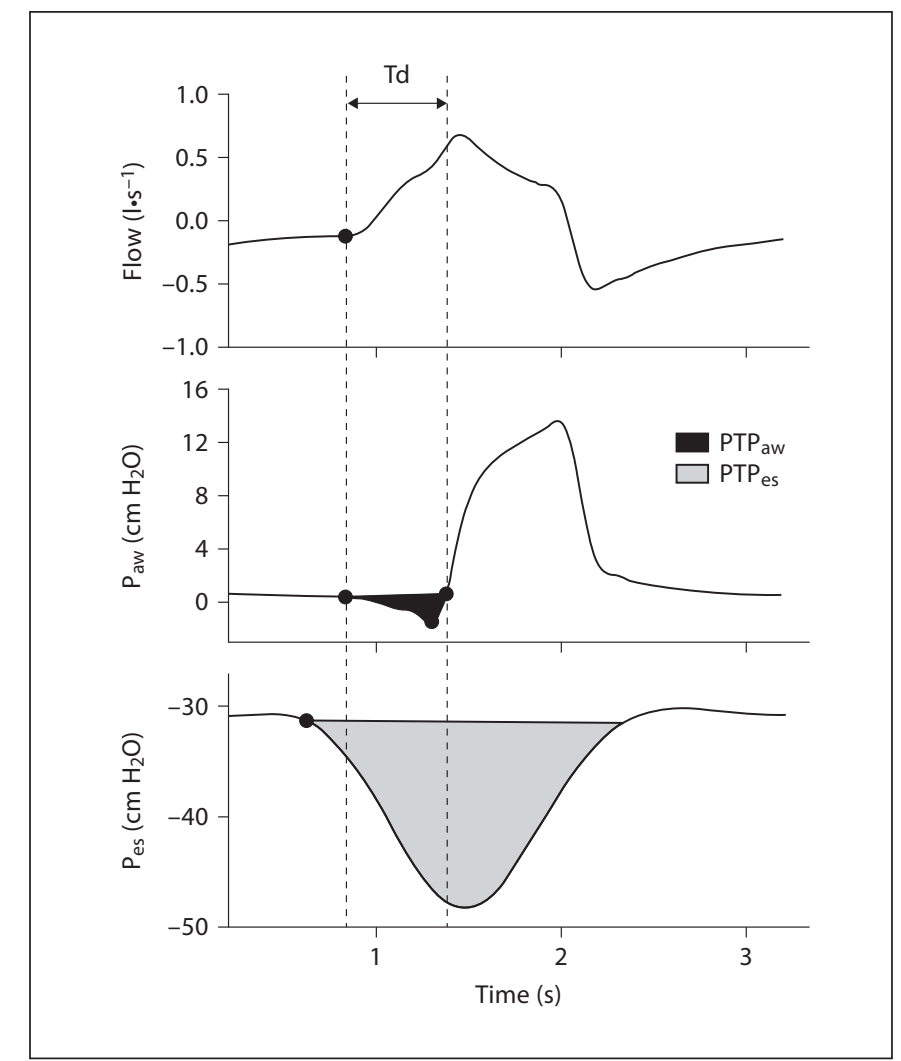

Fig. 1. Representative ventilatory cycle during PSV in 1 patient. Flow (upper panel), $\mathrm{P}_{\mathrm{aw}}$ (middle panel) and $\mathrm{P}_{\mathrm{es}}$ (lower panel) are shown. The $\mathrm{PTP}_{\mathrm{es}}$ was calculated as the area under the $\mathrm{P}_{\mathrm{es}}$ signal from the onset of the negative deflection to the return to baseline. The inspiratory $\mathrm{PTP}_{\mathrm{aw}}$ was calculated as the integral of $\mathrm{P}_{\mathrm{aw}}$ during the trigger time delay (Td).

\section{Statistical Analysis}

Results were expressed as means \pm SD. ANOVA was used to compare breathing patterns and indices of inspiratory effort during spontaneous cycles and triggered and nontriggered cycles during PSV. $\mathrm{p}<0.05$ was chosen for statistical significance.

\section{Results}

One tracing during spontaneous breathing (26.3 \pm 17.6 spontaneous cycles/patient) and 1-9 tracings under PSV were analyzed for each patient. Forty-four tracings covering 2,183 cycles under PSV were analyzed.

\section{Global Identification of ITE by the Phase Portraits}

The flow signals from all the tracings recorded under PSV were analyzed using the phase portrait technique, i.e. representing the time evolution of flow as a two-di- 


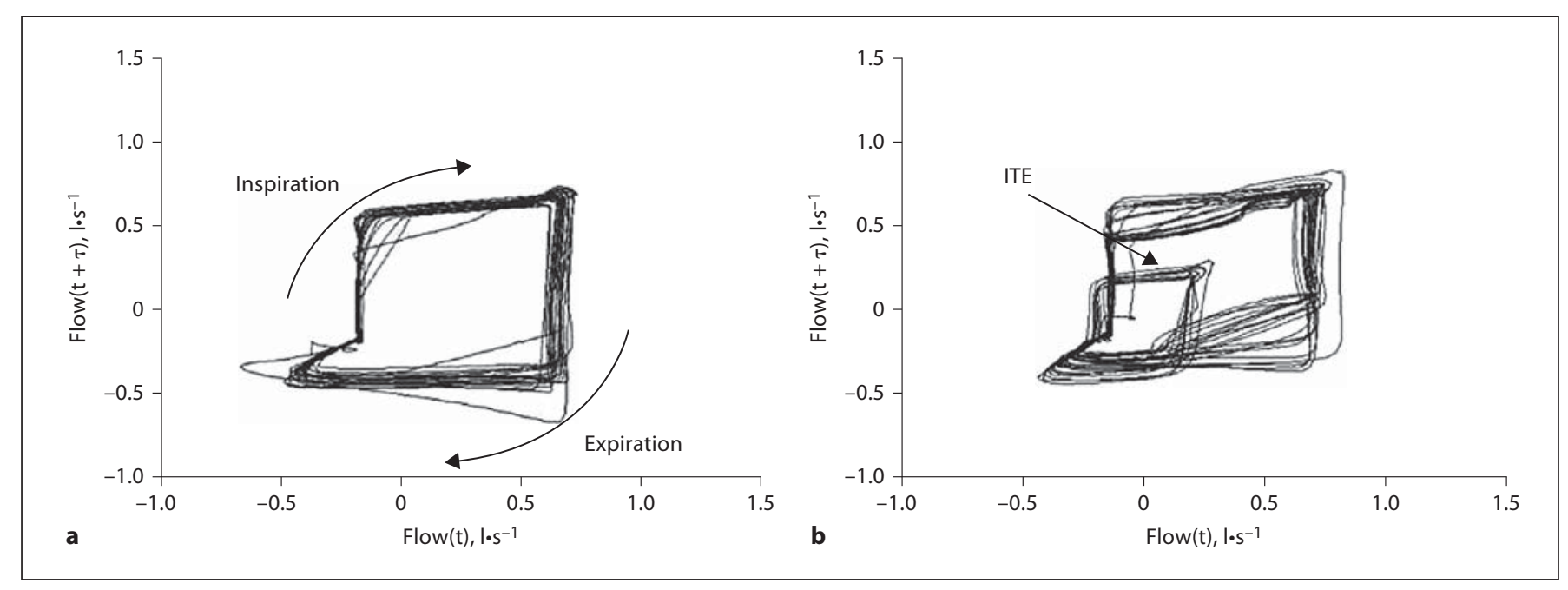

Fig. 2. Phase portraits reconstructed from flow recordings under PSV. Each structure corresponds to the time evolution of flow as a two-dimensional curve in phase space using a time delay $(t+\tau)$ on the corresponding time series as described [16]. a Phase portrait from a representative patient with synchronized and correctly triggered ventilatory cycles. The loops start roughly at the origin $[$ flow $(t)=0$, flow $(t+\tau)=0]$ with the beginning of the in- spiration. A quick jump to an upper plateau corresponds to the rise in pressure. At the end of inspiration, the trajectory switches to a lower plateau associated with expiration, followed by a return to the origin, and so on for further cycles. b Phase portrait from a representative patient with $33 \%$ ineffective triggering efforts. A dichotomous structure with two closed loops is the hallmark of inadequate detection of the inspiratory effort by the ventilator. mensional curve in phase space using a time delay on the corresponding time series (fig. 2). Simple phase portraits were observed in patients with synchronized and correctly triggered cycles during PSV. All respiratory cycles appeared as loops with similar and homogenous morphology (fig. 2a). Phase portraits in patients with ITE were characterized by a dichotomous structure combining two periodic trajectories, that is, larger loops representing triggered ventilatory cycles and smaller loops representing ITE (fig. 2b). Of note, a similar region of the phase space was invariably visited by all the trajectories produced both during triggered and nontriggered cycles. This area corresponded to the end of the expiratory phase when the lungs reached their relaxation volume. Artifacts such as swallowing were associated with a trajectory that greatly differed from the trajectories associated with either triggered cycles or ITE (fig. 3a). Moreover, it was possible to identify the presence of unintentional leaks in very few cycles. When these leaks occurred in the ventilatory circuit, the relationships between the flow $\mathrm{Q}(\mathrm{t})$ at time $t$ and the flow $Q(t+\tau)$ at time $t+\tau$ were very different from those cycles without leaks (fig. 3b). Unintentional leaks were associated with loops that had a significantly higher amplitude than those observed without leaks.

\section{Manual versus Automatic Detection of ITE}

All the ventilatory tracings were manually analyzed in an independent manner by two of the authors (L.A. and C.L.) and classified as a triggered cycle or ITE based on the concomitant $\mathrm{P}_{\mathrm{es}}$ variation. Fifty-six (2.6\%) of the 2,183 ventilatory cycles were ITE and were present in 9 tracings from 4 patients.

Thereafter, the algorithm was used to analyze automatically the flow and $\mathrm{P}_{\mathrm{aw}}$ tracings, without using the $\mathrm{P}_{\mathrm{es}}$ tracings. Table 1 shows the ability of the algorithm to detect ventilatory cycles compared to the manual identification. All the triggered cycles were correctly identified by the algorithm, which also detected 53/56 ITE (94.6\%). Concerning the 3 undetected ITE, the algorithm failed to identify the onset of these breathing cycles because the inspiratory flow changes were barely detectable (fig. 4). Although a significant effort was observed with the $P_{e s}$, the induced flow rate of change was less than the threshold value used to identify the respiratory cycle. The ventilatory cycle was therefore missed and the ITE was consequently not identified (fig. 4a). The corresponding phase portrait, showing a distinct pattern, is shown in figure $4 \mathrm{~b}$. A posteriori attempts to reduce the flow threshold decreased the specificity of the cycle detection by the algorithm (data not shown). 


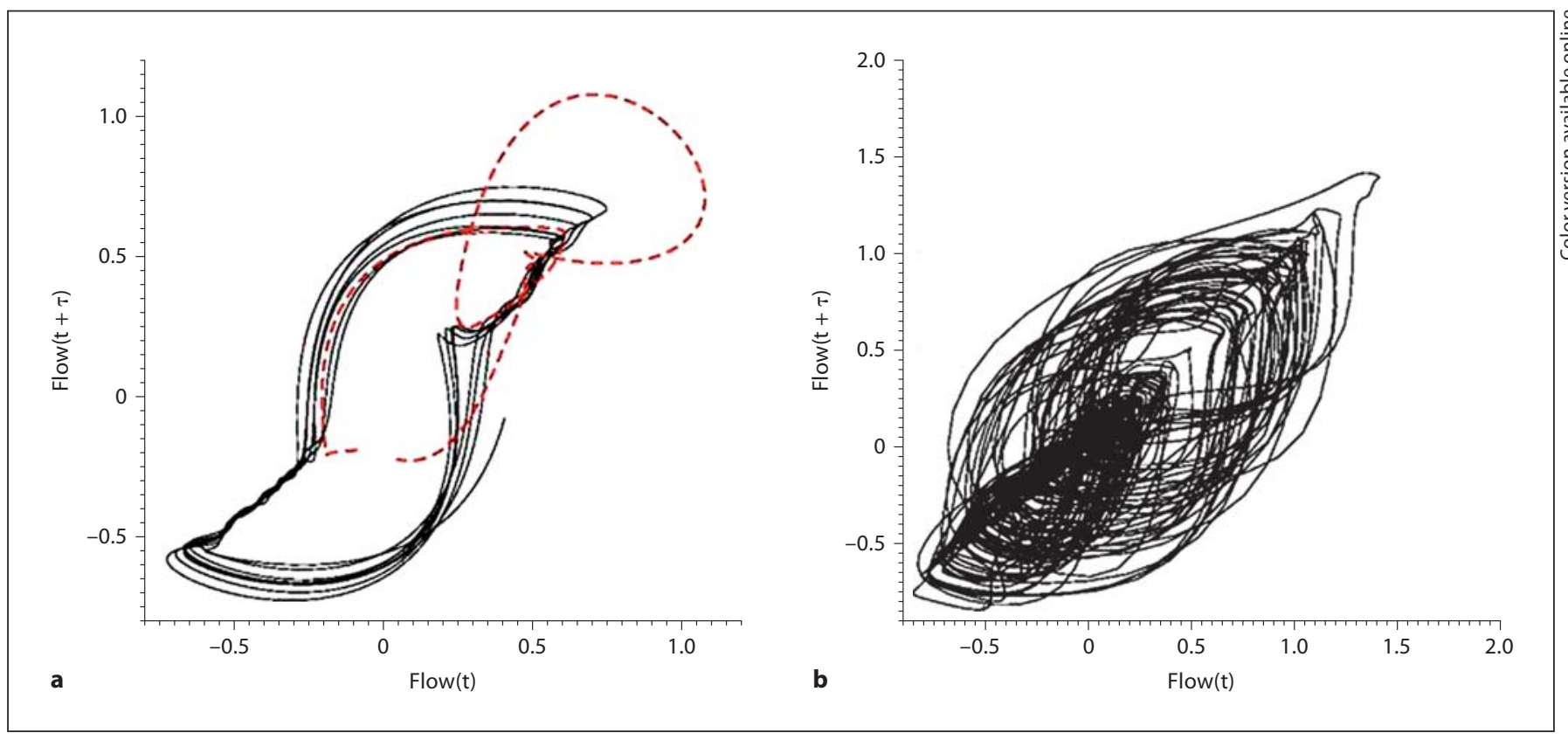

Fig. 3. a Phase portrait from a representative patient with synchronized and correctly triggered cycles (-) associated with a swallowing event (----). b Example of a phase portrait obtained in one child ventilating with significant unintentional leaks, which was recorded for this purpose. The phase portrait is globally shifted along the first diagonal toward higher airflow values. Diverse patient-ventilator interactions were therefore clearly associated with dissimilar trajectories and curve shapes.

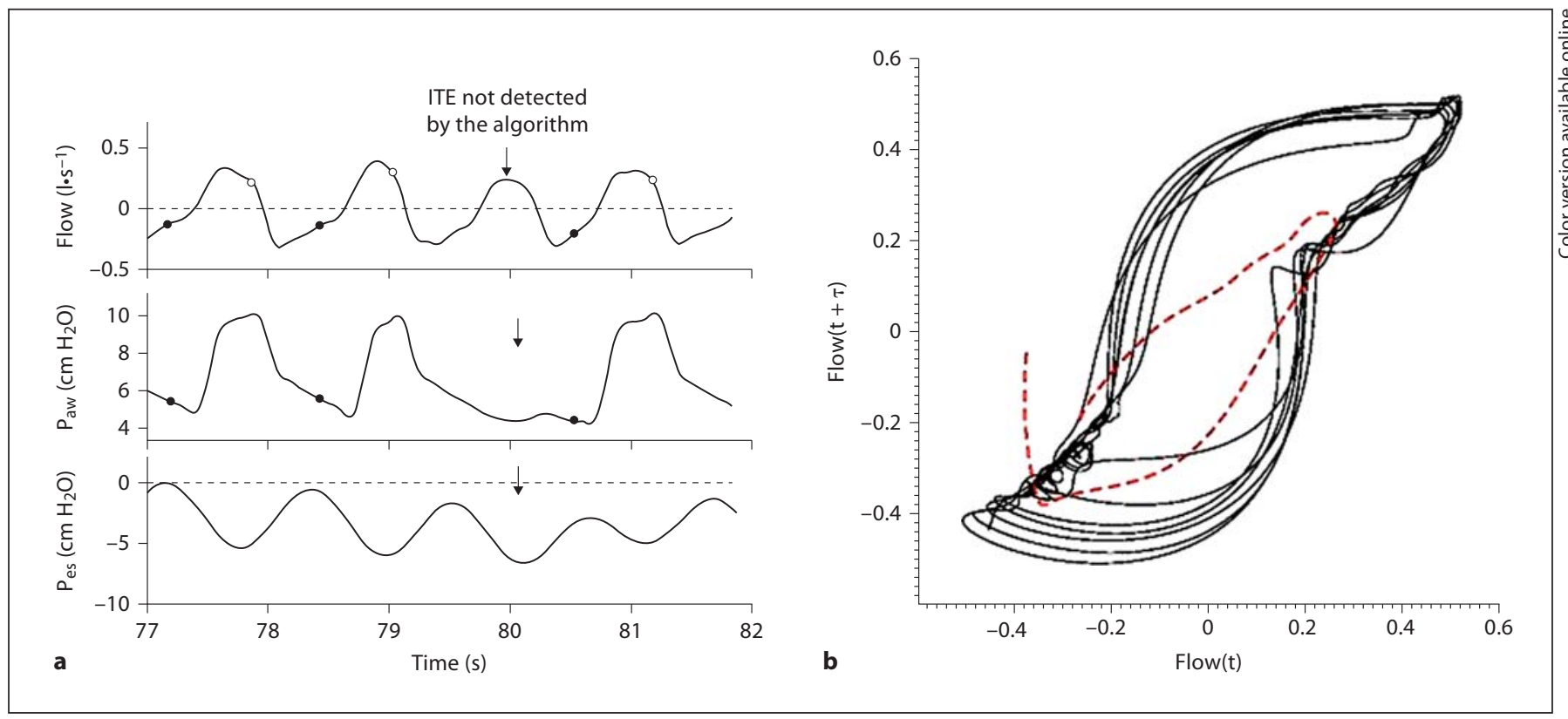

Fig. 4. Ventilatory tracing illustrating one of the three ineffective triggering efforts (arrow) identified by visual analysis but not recognized by the algorithm. Although a significant effort was observed with the $\mathrm{P}_{\mathrm{es}}$, the induced flow variation rate of change was less than the threshold value used by the algorithm to identify the respiratory cycle. The ventilatory cycle was therefore missed and the ITE was consequently not identified (a). $\cdot$ = Onset of mechanical cycle as determined by the algorithm; $\bigcirc=$ onset of expiratory cycling as determined by the algorithm. All the three undetected ITE were of a similar morphology, either on the flow/time or pressure/time series and also the phase portraits that showed a distinct pattern with dotted lines (b; ----). 


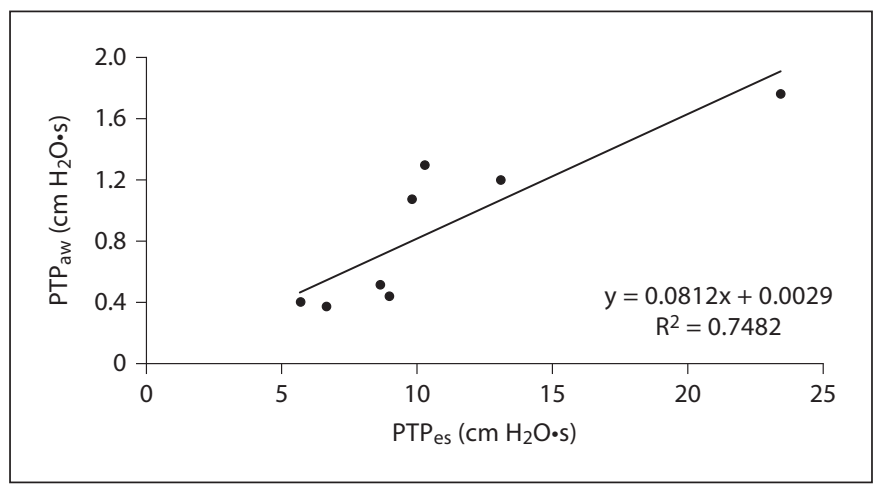

Fig. 5. Correlation between $\mathrm{PTP}_{\mathrm{es}} /$ breath and $\mathrm{PTP}_{\mathrm{aw}} /$ breath during ineffective triggering efforts. $\mathrm{PTP}_{\mathrm{aw}}$ was computed as the integral of $\mathrm{P}_{\mathrm{aw}}$ during the trigger time delay.

Table 1. Ability of the algorithm to detect ventilatory cycles and ineffective triggering efforts compared to the reference method (visual identification by two independent observers)

\begin{tabular}{lcc}
\hline Automatic detection & \multicolumn{2}{c}{ Manual detection } \\
\cline { 2 - 3 } & $\begin{array}{l}\text { triggered cycles } \\
(\mathrm{n}=2,127)\end{array}$ & $\begin{array}{l}\text { ITE } \\
(\mathrm{n}=56)\end{array}$ \\
\hline Triggered cycles $(\mathrm{n}=2,127)$ & 2,127 & 0 \\
ITE $(\mathrm{n}=53)$ & 0 & 53 \\
Undetected cycles $(\mathrm{n}=3)$ & 0 & 3 \\
\hline
\end{tabular}

\section{Consequences of ITE on Breathing Patterns and}

Respiratory Muscle Output

Table 2 shows the breathing patterns of the patients during spontaneous breathing and PSV. As expected, PSV was associated with a significant improvement in VT and VT/Ti compared to spontaneous breathing. When triggered and nontriggered cycles were compared, ITE were associated with a significantly lower VT, but also a lower VE and a 1.7-fold increase in RR ( $\mathrm{p}<0.0001)$ when extrapolating values from all individual cycles to $1 \mathrm{~min}$. TI did not change during ITE, but $\mathrm{TI} / \mathrm{T}_{\text {tot }}$ increased significantly because of a 2 -fold decrease in $\mathrm{Te}(\mathrm{p}<0.0001)$. Lastly, ITE were associated with a 3 -fold decrease in the inspiratory flow compared to triggered cycles.

Our algorithm was also able to analyze the impact of ITE on respiratory muscle output (table 3). As expected, PSV was associated with a significant decrease in respiratory muscle output compared to spontaneous breathing. However, the respiratory muscle output during nontrig- gered cycles was comparable to those measured during spontaneous breathing. $\mathrm{PTP}_{\mathrm{aw}}$ quantification by the automatic method also revealed a significant 2.6 -fold increase in $\mathrm{PTP}_{\mathrm{aw}}$ during ITE compared to synchronized cycles. A significant positive linear correlation was observed between $\mathrm{PTP}_{\mathrm{es}}$ and $\mathrm{PTP}_{\mathrm{aw}}$ during ITE (fig. 5). It was not possible to correlate $\mathrm{PTP}_{\mathrm{es}}$ and $\mathrm{PTP}_{\mathrm{aw}}$ during triggered cycles because minor $\mathrm{P}_{\mathrm{aw}}$ variations were occasionally very close to the noise level.

\section{Discussion}

Our study showed that ITE can be reliably, automatically and noninvasively identified during noninvasive PSV using an algorithm that simultaneously computes flow and $\mathrm{P}_{\mathrm{aw}}$. This detection methodology, based on simple physiological data, is very sensitive and specific as compared to the standard method, that is, measurements of $\mathrm{P}_{\mathrm{es}}$ variations. Also, the structure of the phase portraits reconstructed from the flow time series appeared to be very sensitive to the dynamics of underlying patient-ventilator interactions [16]. The geometric representation under PSV makes it possible to visualize ITE in real time [16]. Phase portraits over a 5-min window provided global signatures of the quality of the ventilation from a mechanical point of view. Depending on loop dispersion and shape, patients with regular ventilatory patterns could be easily distinguished from patients not adapted to their ventilators. It should be noted that air leaks were easily detected by this approach since they induced loops with abnormal shapes and usually with large amplitudes.

Recently, a parallel approach was developed by Mulqueeny et al. [18]. They built an algorithm that was able to detect ineffective triggering efforts based on significant alterations of the expiratory flow signal. However, their technology was connected to a specific ventilator and required an electronic signal delivered by this machine in order to detect the expiratory phase [18]. Conversely, our algorithm runs with any noninvasive ventilator and has the ability to combine the analysis of flow/ pressure time series and their mathematical transformation according to the nonlinear dynamical system theory [16]. Moreover, our algorithm quantifies the breathing pattern and also identifies significant inspiratory effort variations. Chen et al. [19] have finalized an algorithm that works by analyzing minimal and maximal flow values during the expiratory phase of the ITE. By comparison, our algorithm uses flow derivates over each whole 
Table 2. Breathing patterns during spontaneous breathing and pressure support ventilation with the effect of triggered and nontriggered cycles

\begin{tabular}{|c|c|c|c|c|}
\hline & \multirow{2}{*}{$\frac{\text { Spontaneous breathing }}{\text { all cycles }(\mathrm{n}=316)}$} & \multicolumn{3}{|l|}{ PSV } \\
\hline & & all cycles $(\mathrm{n}=2,180)$ & triggered cycles $(\mathrm{n}=2,127)$ & nontriggered cycles $(n=53)$ \\
\hline $\mathrm{RR}, \min ^{-1}$ & $26.5 \pm 8.1$ & $24.4 \pm 6.6^{+}$ & $24.1 \pm 6.5^{+}$ & $40.0 \pm 9.4^{* *}$ \\
\hline $\mathrm{VT}, 1$ & $0.5 \pm 0.1$ & $0.6 \pm 0.2^{+, *}$ & $0.6 \pm 0.2^{+, *}$ & $0.2 \pm 0.1^{*}$ \\
\hline $\mathrm{VE}, 1 / \mathrm{min}$ & $12.1 \pm 4.3$ & $13.4 \pm 3.3^{+}$ & $13.7 \pm 3.2^{+}$ & $6.4 \pm 2.0^{*}$ \\
\hline TI, s & $1.2 \pm 0.2$ & $1.1 \pm 0.3$ & $1.1 \pm 0.3$ & $0.9 \pm 0.2^{*}$ \\
\hline $\mathrm{TE}, \mathrm{s}$ & $1.3 \pm 0.5$ & $1.6 \pm 0.6^{+}$ & $1.7 \pm 0.5^{+, *}$ & $0.8 \pm 0.4^{*}$ \\
\hline $\mathrm{TI} / \mathrm{T}_{\text {tot }}, \%$ & $48.5 \pm 6.7$ & $40.2 \pm 6.0^{+, *}$ & $39.7 \pm 5.7^{+, *}$ & $57.6 \pm 11.1^{*}$ \\
\hline VT/TI, l/s & $0.4 \pm 0.1$ & $0.6 \pm 0.1^{+, * *}$ & $0.6 \pm 0.1^{+, * *}$ & $0.2 \pm 0.0^{*}$ \\
\hline
\end{tabular}

Because nontriggered cycles frequently occur as isolated events and in order to compare the data between each category of cycles, the displayed mean RR and VE values are values of all individual cycles extrapolated to $1 \mathrm{~min} .{ }^{+} \mathrm{p} \leq 0.0001$, significantly different from nontriggered cycles; ${ }^{*} \mathrm{p}<0.05,{ }^{*} \mathrm{p}<0.0001$, significantly different from spontaneous breathing. $\mathrm{RR}=\mathrm{Respiratory}$ rate; VT $=$ tidal volume; $\mathrm{VE}=$ ventilatory minute; $\mathrm{TI}=$ inspiratory time; $\mathrm{TE}=$ expiratory time; $\mathrm{T}_{\text {tot }}=$ total time of the breathing cycle.

Table 3. Impact of triggered cycles and ineffective triggering efforts on respiratory muscle output during pressure support ventilation compared to spontaneous breathing

\begin{tabular}{|c|c|c|c|c|}
\hline & \multirow{2}{*}{$\frac{\text { Spontaneous breathing }}{\text { all cycles }(\mathrm{n}=316)}$} & \multicolumn{3}{|l|}{ PSV } \\
\hline & & all cycles $(n=2,180)$ & triggered cycles $(\mathrm{n}=2,127)$ & nontriggered cycles $(n=53)$ \\
\hline$\Delta \mathrm{P}_{\mathrm{es}}, \mathrm{cm} \mathrm{H}_{2} \mathrm{O}$ & $12.2 \pm 4.1$ & $6.9 \pm 4.3^{+, *}$ & $6.7 \pm 4.2^{+, *}$ & $11.5 \pm 4.7$ \\
\hline $\mathrm{PTP}_{\mathrm{es}} /$ breath, $\mathrm{cm} \mathrm{H}_{2} \mathrm{O} \cdot \mathrm{s}$ & $12.5 \pm 5.1$ & $7.0 \pm 5.4^{+, *}$ & $6.8 \pm 5.4^{+, *}$ & $12.4 \pm 7.0$ \\
\hline $\mathrm{PTP}_{\mathrm{aw}} /$ breath, $\mathrm{cm} \mathrm{H} \mathrm{H}_{2} \mathrm{O} \cdot \mathrm{s}$ & NA & $0.3 \pm 0.7^{*}$ & $0.3 \pm 0.7^{*}$ & $0.8 \pm 0.5$ \\
\hline
\end{tabular}

${ }^{*} \mathrm{p} \leq 0.05$, significantly different from nontriggered cycles; ${ }^{+} \mathrm{p}<0.05$, significantly different from spontaneous breathing.

ventilatory cycle and not only during its sole inspiratory or expiratory phase. We think this approach is therefore very efficient to identify repeated ITE.

The automatic analysis makes it possible to explore the impact of repeated ITE on respiratory patterns. ITE were associated with a 2-fold decrease in VE, a 2-fold decrease in inspiratory flow, a dramatic reduction in VT and a subsequent 1.7-fold increase in RR, compared to triggered cycles when extrapolating values from all individual cycles to $1 \mathrm{~min}$. As expected, recurrent ITE significantly altered the ventilatory pattern under PSV and were associated with decreased alveolar ventilation. In our approach, ITE can be easily distinguished from inspiratory efforts occurring during leakage ventilation because phase portraits during leakage ventilation have a different morphology [16]. Indeed, when using these ventila- tors, leakage ventilation is always associated with higher flows and lower pressures [20, 21].

The automatic analysis also provides an efficient noninvasive methodology to assess the impact of ITE on inspiratory efforts. In our study, $\mathrm{PTP}_{\mathrm{aw}}$ /breath was approximately 2.6-fold higher during ITE breaths than triggered cycles, while inspiratory work indices calculated from $\mathrm{P}_{\mathrm{aw}}$ correlated significantly with those calculated from $\mathrm{P}_{\mathrm{es}}$. The computing of $\mathrm{PTP}_{\mathrm{aw}}$ may thus be a semiquantitative noninvasive tool for quantifying the excessive workload associated with ITE. Conversely, PTP $_{\text {aw }}$ could not be used to evaluate inspiratory work associated with successfully triggered cycles because they only induced minor $\mathrm{P}_{\mathrm{aw}}$ variations that were below the resolution capacity of the data acquisition system. Lastly, our study showed that indices of inspiratory efforts like $\mathrm{P}_{\mathrm{es}}$ swings or $\mathrm{PTP}_{\mathrm{es}} /$ 
breath were similar during ITE and spontaneous breathing, indicating that ITE may reduce the physiological benefits of PSV.

The standard criteria commonly used to define ITE are based on a $\mathrm{P}_{\mathrm{es}}$ decrease $>1 \mathrm{~cm} \mathrm{H}_{2} \mathrm{O}$, with a simultaneous drop in $\mathrm{P}_{\mathrm{aw}}$ and/or change in flow [8]. Giannouli et al. [9] were the first to suggest that ITE could be detected exclusively from $\mathrm{P}_{\mathrm{aw}}$ and flow time series. This approach is accurate in intubated patients and an acceptable ITE underestimation may be encountered in case of severe patient-ventilator asynchronies [9]. Our study showed that a similar approach can be automated and can also be applied to patients receiving noninvasive PSV. Our algorithm could therefore be of potential clinical interest to identify ITE during prolonged recordings during sleep or when assessing domiciliary ventilation, for instance. Moreover, it could also assess breathing patterns and evaluate the respiratory muscle output during each ventilatory cycle. This new approach for assessing patientventilator interactions avoids the inherent difficulties and discomfort associated with $\mathrm{P}_{\mathrm{es}}$ assessments.

Our study had some limitations due to its physiological design. The pneumotachograph was located close to the mask and thus, the algorithm should be further evaluated in the home setting. However, the present study showed that our approach functions in a hospital setting and provides real-time information. The current version of the algorithm cannot identify other types of poor patient-ventilator interactions (double triggering, auto- triggering), but ITE are more prevalent events than these ones [22]. ITE prevalence in our study was similar to other comparable studies $[7,23]$, and we feel that we had a sufficiently large number of ITE to provide accurate characteristics of our algorithm. On the other hand, this ITE rate may be considered as low for a validation study but the relevant point is that almost all ITEs were detected and that the algorithm performance does not depend on the rate of ITE. This was checked by our team in the context of other patients who presented an ITE prevalence $>40 \%$ [24]. Patients in this study were ventilated at home without PEEP because intrinsic PEEP is rather low in CF patients in a stable state [22]. Therefore, the algorithm did function in our study with a PEEP value at 0 , but it has been written and effectively works with a range of PEEP values from 0 to $15 \mathrm{~cm} \mathrm{H}_{2} \mathrm{O}$.

In conclusion, the simultaneous analysis of flow and $\mathrm{P}_{\mathrm{aw}}$ using a dedicated algorithm can reliably identify and quantify ITE during noninvasive PSV without the constraint of $\mathrm{P}_{\mathrm{es}}$ measurements. Moreover, the automatic detection approach can also reliably estimate the excessive inspiratory work associated with ITE by analyzing pressure time series [20].

\section{Acknowledgment}

The authors are grateful to Richard Medeiros (Rouen University Hospital Medical Editor) for his valuable help in editing the manuscript.

\section{References}

1 Fauroux B, Le Roux E, Ravilly S, et al: Longterm noninvasive ventilation in patients with cystic fibrosis. Respiration 2008;76: 168-174.

$\checkmark 2$ Fauroux B, Nicot F, Essouri S, et al: Setting of noninvasive pressure support in young patients with cystic fibrosis. Eur Respir J 2004; 24:624-630.

$\checkmark 3$ Krieger BP: Hyperinflation and intrinsic positive end-expiratory pressure: less room to breathe. Respiration 2009;77:344-350.

4 Nava S, Bruschi C, Rubini F, et al: Respiratory response and inspiratory effort during pressure support ventilation in COPD patients. Intensive Care Med 1995;21:871-879.

5 Nava S, Bruschi C, Fracchia C, et al: Patientventilator interaction and inspiratory effort during pressure support ventilation in patients with different pathologies. Eur Respir J 1997; 10:177-183.
Parthasarathy S: Effects of sleep on patientventilator interaction. Respir Care Clin N Am 2005;11:295-305.

7 Fanfulla F, Delmastro M, Berardinelli A, et al: Effects of different ventilator settings on sleep and inspiratory effort in patients with neuromuscular disease. Am J Respir Crit Care Med 2005;172:619-624.

8 Leung P, Jubran A, Tobin MJ: Comparison of assisted ventilator modes on triggering, patient effort, and dyspnea. Am J Respir Crit Care Med 1997;155:1940-1948.

$\checkmark 9$ Giannouli E, Webster K, Roberts D, et al: Response of ventilator-dependent patients to different levels of pressure support and proportional assist. Am J Respir Crit Care Med 1999;159:1716-1725.

10 Fanfulla F, Taurino AE, Lupo ND, et al: Effect of sleep on patient/ventilator asynchrony in patients undergoing chronic non-invasive mechanical ventilation. Respir Med 2007;101:1702-1707.
11 Guo YF: Contribution of polygraphy and polysomnography to nocturnal monitoring of patients with obesity-hypoventilation syndrome (OHS) and non-invasive ventilation (NIV); medical thesis, University of Geneva, 2004.

12 Parthasarathy S, Jubran A, Tobin MJ: Assessment of neural inspiratory time in ventilator-supported patients. Am J Respir Crit Care Med 2000;162:546-552.

13 Fauroux B, Pigeot J, Polkey MI, et al: In vivo physiologic comparison of two ventilators used for domiciliary ventilation in children with cystic fibrosis. Crit Care Med 2001;29: 2097-2105.

14 Fauroux B, Louis B, Hart N, et al: The effect of back-up rate during non-invasive ventilation in young patients with cystic fibrosis. Intensive Care Med 2004;30:673-681. 
-15 Baydur A, Behrakis PK, Zin WA, et al: A simple method for assessing the validity of the esophageal balloon technique. Am Rev Respir Dis 1982;126:788-791.

16 Achour L, Letellier C, Cuvelier A, et al: Asynchrony and cyclic variability in pressure support non-invasive ventilation. Comput Biol Med 2007;37:1308-1320

-17 Packard NH, Crutchfield JP, Farmer JD, et al: Geometry from a time series. Phys Rev Lett 1980;45:712-716.

$\checkmark 18$ Mulqueeny Q, Ceriana P, Carlucci A, et al: Automatic detection of ineffective triggering and double triggering during mechanical ventilation. Intensive Care Med 2007;33: 2014-2018.
19 Chen CW, Lin WC, Hsu CH, et al: Detecting ineffective triggering in the expiratory phase in mechanically ventilated patients based on airway flow and pressure deflection: feasibility of using a computer algorithm. Crit Care Med 2008;36:455-461.

20 Nilsestuen JO, Hargett KD: Using ventilator graphics to identify patient-ventilator asynchrony. Respir Care 2005;50:202-234.

21 Georgopoulos D, Prinianakis G, Kondili E: Bedside waveforms interpretation as a tool to identify patient-ventilator asynchronies. Intensive Care Med 2006;32:34-47.
22 Thille AW, Rodriguez P, Cabello B, et al: Patient-ventilator asynchrony during assisted mechanical ventilation. Intensive Care Med 2006;32:1515-1522.

23 Vitacca M, Barbano L, D’Anna S, et al: Comparison of five bilevel pressure ventilators in patients with chronic ventilatory failure: a physiologic study. Chest 2002;122:21052114.

24 Rabarimanantsoa H: Qualité des interactions patient-ventilateur en ventilation non invasive nocturne; $\mathrm{PhD}$ thesis, University of Rouen, 2008. www.coria.fr/spip.php?article551. 